\title{
Metabolic heterogeneity and immunocompetence of infiltrating immune cells in the breast cancer microenvironment (Review)
}

\author{
HONGDAN CHEN*, YIZENG SUN*, ZEYU YANG, SUPENG YIN, YAO LI, \\ MI TANG, JUNPING ZHU and FAN ZHANG \\ Department of Breast and Thyroid Surgery, Chongqing General Hospital, \\ University of Chinese Academy of Sciences, Chongqing 401147, P.R. China
}

Received September 3, 2020; Accepted January 8, 2021

DOI: $10.3892 /$ or.2021.7946

\begin{abstract}
Breast cancer is one of the most common malignancies in women and is characterized by active immunogenicity. Immune cell infiltration plays an important role in the development of breast cancer. The degree of infiltration influences both the response to and effect of treatment. However, immune infiltration is a complex process. Differences in oxygen partial pressure, blood perfusion and nutrients in the tumor microenvironment (TME) suggest that infiltrating immune cells in different sites experience different microenvironments with corresponding changes in the metabolic mode, that is, immune cell metabolism is heterogenous in the TME. Furthermore, the present review found that lipid metabolism can support the immunosuppressive microenvironment in breast cancer based on a review of published literature. Research in this field is still ongoing; however, it is vital to understand the metabolic patterns and effects of different microenvironments for antitumor therapy. Therefore, this review discusses the metabolic responses of various immune cells to different microenvironments in breast cancer and provides potentially meaningful insights for tumor immunotherapy.
\end{abstract}

\section{Contents}

1. Introduction

2. Relationship between metabolism, tumors and immune cells

3. Lymphocytes

Correspondence to: Professor Fan Zhang, Department of Breast and Thyroid Surgery, Chongqing General Hospital, University of Chinese Academy of Sciences, 118 Xingguang Avenue, Chongqing 401147, P.R. China

E-mail: zhangfancgh@163.com

*Contributed equally

Key words: breast cancer, tumor microenvironment, immune infiltration, metabolic heterogeneity
4. Macrophages
5. Natural killer (NK) cells
6. DCs
7. Other immune cells
8. Conclusions

\section{Introduction}

Breast cancer is one of the most common malignant tumors in women (1). Research has demonstrated that breast cancer has certain immunogenicity (2). The types, degree and related phenotypes of immune cells infiltrating the tumor microenvironment (TME) are closely related to the prognosis of patients (3-5). However, almost all aspects of the immune response, such as the maturation and antigen presentation capacity of dendritic cells (DCs) and the infiltration and differentiation of immune cells, are closely related to the metabolic patterns of these cells and surrounding cells $(6,7)$. The TME changes along a relatively smooth gradient, from a well-perfused perivascular area to an ischemic area, suggesting that immune cells will experience a variety of different microenvironments (8), which may vary from nutrient-rich, oxygen-sufficient sites to low-oxygen sites with competition for nutrients and even to hypoxic, nutrient-deprived sites or sites with metabolic waste accumulation (Fig. 1); this variation may also be a useful explanation for the early suppression of antitumor immunity. At the same time, it also leads to the metabolic heterogeneity of immune cells in the TME, which creates challenges for tumor immunotherapy.

Since Otto Warburg proposed in 1924 that tumor cells tend to produce energy rapidly through glycolysis (9), the important role of metabolic reprogramming in tumor development has gradually been recognized $(10,11)$. However, the importance of lipid metabolism was generally ignored over the years, but lipid metabolism has been widely studied in the past few years. Based on previous research, we speculate that changes in lipid metabolism, especially fatty acid metabolism, are crucial in determining the immune activity or tolerance of immune cells. The metabolic pattern of infiltrating immune cells in breast cancer changes significantly across different environments. However, at present, research on this topic is not cohesive. Therefore, this review focuses on the metabolic changes 
in several immune cells with relatively high infiltration in different microenvironments in breast cancer and addresses how cells are changed into a 'bystander' or an 'accomplice' by regulating lipid metabolism. It is helpful to further understand the metabolic heterogeneity of infiltrating immune cells in breast cancer in the contexts of different backgrounds and provide novel ideas for immunotherapy. Abbreviations used in the present review are included in Table I.

\section{Relationship between metabolism, tumors and immune cells}

To understand the metabolic heterogeneity of immune cells in breast cancer, the relationship among tumor cells, immune cells and metabolism was analyzed in this review. Every aspect of tumor tissue that differs from that of normal tissue may be the cause of tumor metabolic heterogeneity. In recent years, an increasing number of studies have shown that the metabolic patterns of different groups in tumors are coupled with each other through metabolism, and metabolism plays a very important role in the functional maintenance and directional differentiation of immune cells; that is, immune cells in the TME will withstand metabolic reprogramming, undergoing either activation to play an antitumor role or immune tolerance to promote tumor progression (12-15).

Metabolic heterogeneity is one of the markers of breast cancer, and the genetic and phenotypic diversity of breast cancer is the main obstacle when treating tumors (16). Due to random genetic changes, intratumoral heterogeneity is generally considered to be chaotic. By contrast, changes in tumor cell metabolism produces predictable extracellular metabolite gradients, which combined with the distance of the tumor cells from the blood supply (17), forms a unique TME and thus coordinates the diverse phenotypes of various cells $(18,19)$. Previous studies have shown that breast cancer cells have a higher extracellular environment acidification capacity than normal breast cells $(20,21)$. On the other hand, the rapid growth of cells is faster than the rate of capillary formation in cancer, leading to gradual hypoxia in breast cancer tissue $(22,23)$, and creating a nutrient-deficient environment (24). The series of gradient changes described above leads to immune cells making metabolic adjustments that correspond to these different microenvironments. The present review described the changes in the metabolic patterns of tumor-infiltrating immune cells in different microenvironments and the induction of a tolerance phenotype by lipid metabolism.

\section{Lymphocytes}

Tumor-infiltrating lymphocytes (TILs) have a strong prognostic value in various types of cancer (25), and they are also the most common type of infiltrating immune cell in breast cancer (26). T cells comprise heterogeneous cell groups with a wide range of effector mechanisms, ranging from immunosuppression to cytotoxicity.

Metabolic characteristics of T lymphocyte subtypes. T lymphocytes are activated to become mature $\mathrm{T}$ lymphocytes that show associated functions via stimulation of $\mathrm{T}$ cell receptor (TCR) signaling. Activated T cells signifi- cantly upregulate glycolysis and lactate production (27). ADP-dependent glucokinase (ADPGK), which is a protein typically found in Archaea whose function in eukaryotes was unknown, is activated in this process, which is accompanied by rapid glucose uptake and decreased mitochondrial oxygen consumption, thus resulting in the increase of glycolysis flux (28). However, it cannot be ignored that mitochondria-dependent metabolism still plays an important role in the $\mathrm{T}$ cell response (29). In the absence of TCR stimulation, immature $T$ cells remain in a dormant state, reducing the expression of nutrient transporters, but still maintaining certain catabolic processes, including autophagy, oxidative phosphorylation (OXPHOS) and fatty acid oxidation (FAO) (30). According to the literature, different lymphocyte subtypes exhibit different metabolic patterns during the activation process, in which the effector T lymphocytes show high glycolysis and lipogenesis (31), while regulatory $\mathrm{T}$ cells (Tregs) show higher lipolysis and lipid oxidation (32).

Influence of lactic acid and hypoxia on the T cell phenotype. Due to factors such as hypoxia, lactate and adenosine accumulation, nutrient deficiency, and immunosuppression, the activation and survival of $\mathrm{T}$ cells face great challenges. Both normal and tumor cells can adapt to hypoxia or a hypoxic microenvironment by regulating hypoxia inducible factor (HIF). A lack of oxygen supply, or hypoxia, will increase the expression and stability of HIF in T lymphocytes, thus activating certain molecular programs, including glycolysis (33). HIF mainly reduces oxygen consumption by increasing the expression of pyruvate dehydrogenase kinase 1 (PDK1), thus promoting $\mathrm{T}$ cell adaptation to hypoxia (34). At this time, $\mathrm{T}$ lymphocytes also play a corresponding role by regulating their own metabolic mode. However, in the central tumor area with extreme hypoxia, $\mathrm{T}$ cell survival is threatened. To survive, $\mathrm{T}$ cells reduce their dependence on glycolysis, developing an immunosuppressive phenotype (35). Studies have demonstrated that breast cancer tumors secreting a large amount of lactate have relatively great metastatic potential, and the prognosis of patients with these tumors is worse than that of patients with tumors secreting less lactate $(36,37)$. One of the possible reasons is that the acidic environment formed by the accumulation of a large amount of lactate inhibits the proliferation and function of T cells $(38,39)$. Activated T cells also depend on glycolysis. Due to the high demand for energy in the processes of proliferation and cytokine production, to ensure continuous glycolysis, cells pump out lactate molecules. The large accumulation of lactate in the breast cancer environment leads to an inappropriate lactate gradient between the extracellular environment and the cytoplasm, thus reducing energy metabolism and ultimately infiltrated $\mathrm{T}$ cells gradually polarize into the immunosuppressive Treg phenotype (40).

In addition, glucose deprivation increases the ability of T helper (Th) cells to secrete transforming growth factor $\beta$, thus confirming the shift of the microenvironment from immunostimulatory to immunosuppressive (41). It has been reported that $\mathrm{T}$ cells can transition into a metabolic mode to perform lactate uptake in a glucose-deficient TME (42). Besides, the reversal of the lactic dehydrogenase reaction to generate pyruvate depletes nicotinamide adenine dinucleotide and effectively inhibits GAPDH activity and glycolytic flux (43), 
Table I. List of abbreviations in the review.

\begin{tabular}{|c|c|}
\hline Abbreviation & Full name \\
\hline 2-DG & 2-deoxyglucose \\
\hline AMPK & $\begin{array}{l}\text { Adenosine 5'-monophosphate (AMP) } \\
\text {-activated protein kinase }\end{array}$ \\
\hline ARG1 & Arginase 1 \\
\hline ATP & Adenosine triphosphate \\
\hline BMDCs & Bone marrow-derived dendritic cells \\
\hline CCR7 & C-C motif chemokine receptor 7 \\
\hline CD36 & CD36 molecule \\
\hline CD56 & CD56 molecule \\
\hline CD68 & CD68 molecule \\
\hline cDCs & Conventional dendritic cells \\
\hline DCs & Dendritic cells \\
\hline EMT & Epithelial-mesenchymal transition \\
\hline FAO & Fatty acid oxidation \\
\hline FASN & Fatty acid synthetase \\
\hline FATP2 & Fatty acid transporter 2 \\
\hline FATP4 & Fatty acid transporter 4 \\
\hline IL-4 & Interleukin-4 \\
\hline LDL & Low density lipoprotein \\
\hline MDSCs & Myeloid-derived suppressor cells \\
\hline mTOR & Mechanistic target of rapamycin kinas \\
\hline NK cells & Natural killer cells \\
\hline $\mathrm{NO}$ & Nitric oxide \\
\hline OXPHOS & Oxidative phosphorylation \\
\hline PMN-MDSCs & $\begin{array}{l}\text { Polymorphonuclear myeloid-derived } \\
\text { suppressor cells }\end{array}$ \\
\hline Foxp3 & Forkhead box P3 \\
\hline GLUT1 (SLC2A1) & Solute carrier family 2 member 1 \\
\hline HIF & Hypoxia inducible factor \\
\hline IFN- $\gamma$ & Interferon- $\gamma$ \\
\hline IL-12 & Interleukin-12 \\
\hline IL-15 & Interleukin-15 \\
\hline IL-2 & Interleukin-2 \\
\hline TAM & Tumor-associated macrophage \\
\hline TCR & $\mathrm{T}$ cell receptor \\
\hline Th cells & T helper cells \\
\hline TILs & Tumor-infiltrating lymphocytes \\
\hline TME & Tumor microenvironment \\
\hline Tregs & Regulatory T cells \\
\hline VLDL & Very low-density lipoprotein \\
\hline
\end{tabular}

which is particularly harmful to cytotoxicity and effector $\mathrm{T}$ cells. Of note, immunosuppressive Tregs show resistance to lactate inhibition via downregulation of c-Myc expression by forkhead box P3 (Foxp3), which reduces glycolysis dependence (44). At the same time, T cells stimulated by TCR signaling in glutamine- and glucose-deficient conditions preferentially differentiate into Tregs, which may be because their oxidative phenotypes are metabolically suited for survival in this environment (36). When the survival of cells is threatened, their metabolic mode changes, and glucose is no longer the first-choice energy source; instead, cells are more inclined to use lipids for energy supply and maintenance (45). This is because the outer edge of tumor tissue with abundant blood vessels in breast cancer mostly contains effector $\mathrm{T}$ cells, and in the area lacking a sufficient blood supply, Tregs have more advantages; these differences reflect the metabolic heterogeneity of TILs (46).

Contribution of lipid metabolism to the differentiation of $T$ cells into Tregs. Various studies have found that OXPHOS and glycolysis, especially glycolytic flux, are significantly increased in effector T cells (47-49). By contrast, Tregs show a unique metabolic program that mainly utilizes mitochondrial oxidation of lipids and pyruvate $(50,51)$. Other evidence has suggested that inhibition of glycolysis obstructs the development of Th1 and Th17 cells, but promotes the production of Tregs (52). Adenosine 5'-monophosphate (AMP)-activated protein kinase (AMPK) is a major sensor and regulator of energy metabolism in mammalian cells. AMPK interferes with $\mathrm{T}$ cell differentiation and effector functions by suppressing mechanistic target of rapamycin kinase (mTOR) and subsequently inhibiting glycolysis and enhancing lipid oxidation (52).

Foxp3 is highly expressed in Tregs. Studies have reported that Foxp3 plays an important role in the regulation of metabolism $(53,54)$. In a previous study, $\mathrm{CD} 4^{+} \mathrm{T}$ cells were stimulated and transduced to express Foxp3 under neutral conditions, and the results showed that the expression of lipid metabolism-related genes was significantly increased in the Foxp3-expressing cells, while the expression of genes related to glucose and nucleic acid metabolism was downregulated (55). It is speculated that Foxp3 may directly regulate the PI3K-AKT-mTORC1 pathway or indirectly regulate the expression of metabolic genes and establish a phenotype of glycolysis inhibition (55). Immune cells respond to activation and toll-like receptor (TLR) signaling by increasing solute carrier family 2 member 1 (GLUT1) expression and glycolysis (55). By contrast, Foxp3 reduces GLUT1 expression, glycolysis and anabolism, indicating an activated mitochondrial oxidation pathway (56). In vitro studies have also demonstrated that Foxp $3^{+}$Tregs mainly rely on lipid oxidation to promote mitochondrial OXPHOS, and it has been speculated that Foxp3 expression is the basis of this metabolic preference (55).

\section{Macrophages}

Tumor-associated macrophages (TAMs), another 'main force' in the TME, have been observed in the invasive front of breast cancer tumors in patients (57). Previous reports demonstrated that compared with malignant cells that have not undergone epithelial-mesenchymal transition (EMT), breast cancer cells with EMT changes have the ability to polarize macrophages into the M2 phenotype, suggesting that macrophages in the breast cancer microenvironment play an important role in tumor invasion $(58,59)$. As commonly known, the main subtypes of macrophages are proinflammatory M1 macrophages and anti-inflammatory M2 macrophages. M1 macrophages mainly secrete cytokines such as interferon- $\gamma$ (IFN- $\gamma)$, interleukin (IL)- 8 and TNF- $\alpha$, which play pro-inflammatory and 


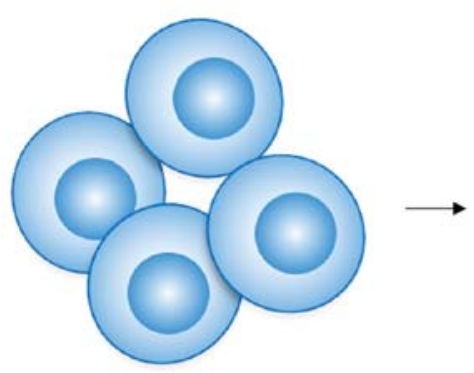

Primary immune cells

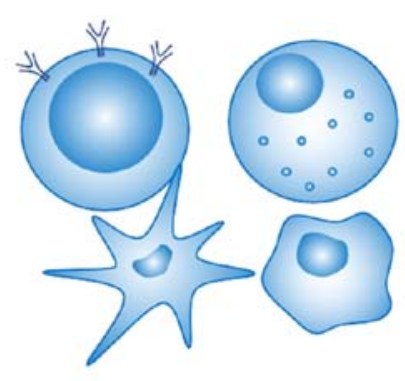

Activated immune cells

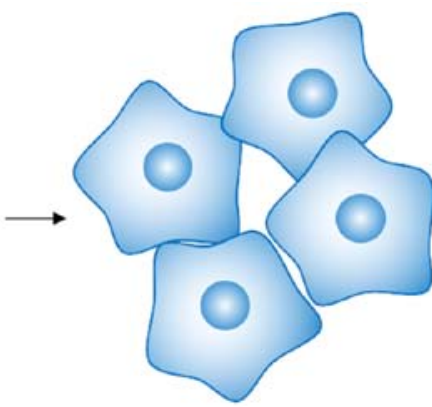

Tolerant immune cells

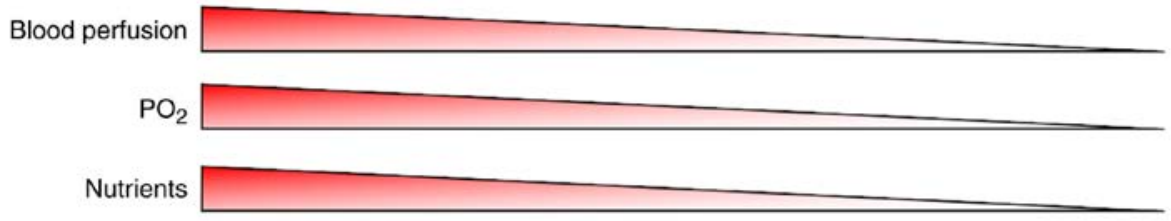

Metabolic wastes

(e.g., lactic acids)

Figure 1. Tumor-infiltrating immune cells experience a complicated microenvironmental challenge. The tumor microenvironment transitions from areas with abundant nutrients and oxygen to those with low oxygen and competition for nutrients or even hypoxia, nutrient deprivation and metabolic waste accumulation, and the state of immune cells changes accordingly. Immune cells are activated from the initial state to become immune cells with different effector functions. When survival is threatened, immune cells develop a resting or tolerant phenotype.

antitumor roles. On the other hand, M2 macrophages mainly secrete factors such as IL-13, C-C motif chemokine (CCL) 17 and CCL18 to promote tumor development $(60,61)$. Due to a combination of numerous factors and the complexity of the TME, the phenotype of TAMs may be between M1 and M2 types, or different from M1 or M2 types that can't be regarded as either type specifically. Thus, TAMs can no longer be simply considered either/or populations (62).

Metabolic characteristics of macrophage subtypes. To clarify the metabolic characteristics of macrophage subtypes, cells can still be divided into M1 and M2 type macrophages. M1 macrophages show enhanced aerobic glycolysis, increased pentose phosphate pathway activity and fatty acid synthesis flux. However, at the level of succinate dehydrogenase and isocitrate dehydrogenase, M1 macrophages also exhibit incomplete OXPHOS, and mitochondrial adenosine triphosphate (ATP) synthesis is blocked (63). M2 macrophages break down arginine into urea and urethane via arginase 1 (ARG1). ARG1 is a representative marker of $\mathrm{M} 2$ macrophages, and nitric oxide (NO) production in M2 macrophages is blocked, resulting in inhibition of nitroso-mediated OXPHOS, which is conducive to maintaining the M2 phenotype (64). M2 macrophages show relatively low levels of glycolysis and enhanced FAO to fuel OXPHOS (65). Highly glycolytic tumor cells may prevent polarization into the M1 phenotype by inducing glucose deprivation, while the abundance of fatty acids may affect the differentiation of cells into the M2 phenotype $(66,67)$.

Influence of lactic acid and hypoxia on the macrophage phenotype. Similar to TILs, tumor-infiltrating macrophages with different spatial distributions face different challenges and respond accordingly. Carmona-Fontaine et al (19) found that TAMs expressing ARG1 were almost completely located in the ischemic tumor area, while TAMs expressing mannose receptor C-type 1 (MRC1) were found in the perivascular and other well-nourished tumor areas, and the research also showed that the subgroup of TAMs expressing MRC1 in the perivascular region of patients with breast cancer was important for tumor recurrence after chemotherapy (19). Some studies have reported that lactate produced by breast cancer cells, a key metabolite in the TME, can promote M2-like polarization of macrophages by inducing high expression of VEGF and ARG1 in macrophages, and this series of changes may be mediated by HIF-1 $\alpha(68,69)$. Almost all studies have provided extensive evidence of the synergistic effect of hypoxia and lactate $(70,71)$. When macrophages in normoxic or hypoxic environments are treated with various lactate doses, the ARG1 protein level in macrophages increases in hypoxic conditions, but not in normoxic conditions (19). Additionally, macrophages activated by lactate and/or hypoxia can induce aerobic glycolysis and epithelial stromal transformation in tumor cells by regulating the CCL5/C-C chemokine receptor type 5 (CCR5) axis, forming a regulatory feedback loop to promote the progression of breast cancer (72). The metabolic pattern of M1 macrophages is similar to that of tumor cells, showing highly activated glycolysis, which indicates that M1 macrophages and tumor cells compete with and suppress each other (73). By contrast, M2 macrophages preferentially use FAO, which is more conducive to their survival in the TME, and became a favorable promoter of tumor progression (74).

Important role of lipid metabolism in the immunosuppressive TAM phenotype. TAMs promote tumor growth and metastasis by inhibiting tumor immune surveillance. There is evidence that the immunosuppressive phenotype of TAMs is regulated by long-chain fatty acid metabolism, especially unsaturated fatty acid metabolism (75). In vitro, the addition of unsaturated 
fatty acids was found to polarize myeloid cells derived from the bone marrow into M2 macrophages with a strong inhibitory ability. Lipid droplets play a vital role by regulating the catabolism of free fatty acids during mitochondrial respiration (76). IL-4-induced M2 macrophages increase their expression of CD36, thus enhancing the uptake of very low-density lipoprotein (VLDL) and LDL, activate FAO, and rely on FAO to support proliferation (77). Inhibition of mTOR eliminates the mitochondrial respiration induced by lipid droplets, thus eliminating the immunosuppressive effect of TAMs (75). A previous study reported that simvastatin repolarizes TAMs and promotes M2 to M1 phenotypic conversion through cholesterol-related liver $\mathrm{X}$ receptor/ATP binding cassette transporter A1 regulation (78). These results suggested that lipid metabolism plays an important role in the differentiation and functional maintenance of M2 macrophages and that further study of lipid metabolism has the potential to identify potential targets and generate novel antitumor treatments.

\section{Natural killer (NK) cells}

NK cells are key components in innate immunity. They are mainly generated from hematopoietic stem cells that develop in the bone marrow and distribute to multiple peripheral tissues after maturation (79). They have the potential to kill tumor cells in different ways without prior sensitization, so they have become an important tool in cancer immunotherapy (80).

Metabolic characteristics of NK cells. In general, resting NK cells use OXPHOS to meet their own steady-state needs because this pathway can effectively generate energy without requiring an excessive investment in synthesis (81). When NK cells are activated, they change their metabolic mode to be able to create the large number of biosynthetic precursors required for the synthesis of effector molecules. NK cells have been proven to be able to strongly upregulate glycolysis and the OXPHOS pathway $(82,83)$. Activated NK cells transform glycolysis-derived NADH into mitochondrial NADH via the citrate-malate shuttle mechanism, which promotes OXPHOS and the synthesis of ATP (84). NK cells are widely characterized as CD56 ${ }^{\mathrm{dim}}$ and CD56 ${ }^{\text {bright }}(85)$. These subsets also differ in terms of metabolism. CD56 $6^{\text {bright }}$ cells are more sensitive to metabolic changes, and their upregulation of the expression of centralized metabolic markers is stronger than that of $\mathrm{CD}^{\mathrm{dim}}$ cells (82). Schafer et al (86) recently reported that NK cells with low reactivity used mitochondrial respiration to activate cytotoxic function, while functional NK cells showed increased glycolysis accompanied by OXPHOS. One of the main limitations of NK cell activity is the immunosuppressive TME. Tumors and other immune cells create conditions that favor tumor proliferation, while also blocking the activation of NK cells.

Influence of lactic acid and hypoxia on the NK cell phenotype. The activity of NK cells is lower near the ischemic area in the tumor center, and it is difficult for even NK cells to infiltrate into the central area. The lack of nutrients and oxygen, and high concentrations of tumor-derived metabolites (such as lactate and adenosine) disrupt the metabolism of NK cells in the TME (80). Short-term exposure to hypoxia enhances the function of NK cells (87), but after long-term hypoxia, NK cells upregulate HIF-1 $\alpha$ expression, resulting in a change in the transcriptional profile and obvious downregulation of the expression of cytotoxic receptors, such as natural killer cell p30-related protein (NKp30), NKp44, NKp46 and natural killer group 2 member D (88). At the same time, HIF-1 $\alpha$ also affects the following: i) Glycolytic enzymes M2 isoform of pyruvate kinase and phosphoglycerate kinase 1 ; ii) the metabolite transporters, including GLUT1, solute carrier family 2 member 3, solute carrier family 1 member 5 and solute carrier family 16 member 4; and iii) enzymes involved in biosynthesis, such as fatty acid synthetase (FASN) and glucose 6-phosphatedehydrogenase (89). The expression of IFN- $\gamma$ in mature NK cells is decreased, the production of IFN- $\gamma$ is decreased, and OXPHOS is reduced (90).

A recent study showed that adenosine attenuated the metabolic activity of IL-12/15-stimulated human NK cells by inhibiting OXPHOS and glycolysis (91). Furthermore, it has also been demonstrated that the uptake of lactic acid by NK cells in the TME leads to intracellular acidification and energy metabolism disruption (92). On the one hand, the increasing demand of tumors for amino acids and the lack of a fuel supply in the microenvironment reduces the functions of NK cells. We also speculated that the consumption of amino acids by tumor cells and tumor-related cells will lead to the accumulation of immunosuppressive metabolites in the TME, which indirectly affects the function of NK cells. For example, myeloid-derived suppressor cells (MDSCs) upregulate the expression of arginase and inducible NO synthetase, which use arginine as a substrate, and the latter metabolizes arginine into NO. It has been found that NO weakens the cytotoxicity of antibody-dependent NK cells (93). NK cells in breast cancer gradually experience an increasingly harsh environment. The metabolism of NK cells is negatively affected, with the cells transitioning from an antitumor function to being unable to undergo activation or even becoming unable to survive in the central tumor area without blood perfusion (94).

Effect of lipid metabolism activation on NK cell immune tolerance. NK cells are significantly inhibited in the severe TME and are even found in the resting state (95). It was found that genes related to glycolysis and OXPHOS were downregulated in NK cells undergoing severe TME. By contrast, the activity of lipid metabolism is increased $(96,97)$. Studies have also confirmed that when NK cells survival is threatened, lipid metabolism becomes the preferred mode of metabolism $(98,99)$. In a mouse model of breast cancer, NK cells appeared to accumulate lipids in vivo, which was mediated by CD36 and CD68, after surgery. These NK cells showed an inhibitory effector function with downregulation of the expression of perforin- and granzyme-related genes (100). Peroxisome proliferator-activated receptor drives lipid accumulation in NK cells, leading to complete 'paralysis' of cell metabolism and transportation (101). Preventing lipids from entering the mitochondria reverses NK cell metabolic paralysis and restores cytotoxicity (101). Compared with immune cells with an immunotolerant phenotype, NK cells have shown a smaller effect on cell function mediated by lipid reprogramming, and the mechanism of action is not sufficiently understood (101). However, it is of great significance and value 
to identify the potential targets of lipid metabolism in NK cells and thereby improve immunotherapy.

\section{DCs}

Tumor-associated DCs have major defects in function and activity, and promote tumor immunosuppression. Studies have reported that abnormal lipid accumulation in DCs is one of the main mechanisms leading to DC dysfunction (102,103). DCs are important regulators of activation or tolerance in the adaptive immune response (104), and are also one of the most important types of antigen-presenting cells in the breast cancer microenvironment (105). DCs can not only regulate immunogenicity, but also induce tolerance. One of the key factors determining this functional fate is the metabolic process of DCs (13).

Metabolic characteristics of DC subtypes. In the process of DC activation, glycolysis and glucose, as the preferred carbon source, can promote an immunogenic or inflammatory state, while OXPHOS and FAO are conducive to the transformation of tolerant DCs $(106,107)$. Glycolysis is important for the maturation and function of both conventional DCs (cDCs) and bone marrow-derived DCs (BMDCs). Treatment with 2-deoxyglucose (2-DG, an inhibitor of glycolysis) impairs the expression of costimulatory markers, the production of IL-12, and functioning by BMDCs and cDCs (108). Glucose can promote the migration of BMDCs and $\mathrm{CDCs}$ along a $\mathrm{C}-\mathrm{C}$ motif chemokine ligand 21 gradient, which can be inhibited by 2-DG treatment (108). In addition, glycolysis is also needed to maintain the slender cell shape of BMDCs and promote CCR7 oligomerization so that DCs can move and migrate to the draining lymph nodes (109).

Influence of lactic acid and hypoxia on the DC phenotype. The duality of DC immunoregulatory functions mainly depend on the differentiation and activation state of DCs (104). DCs undergo metabolic transformation in the process of maturation, from FAO and OXPHOS to glycolysis. Unlike the transformations of tumor cells and effector T cells, this transformation of DCs does not promote cell division, but is crucial in the activation and survival of DCs after TLR stimulation (110). The concentration of lactate after glycolysis, rather than the availability of oxygen tends to shift the differentiation of DCs in the direction of tolerance (111). The high concentration of extracellular lactate in the TME may prevent lactate output from glycolysis-dependent DCs. Tumor-derived lactate is an important factor regulating the DC phenotype in the TME, which may play a key role in the tumor escape mechanism (112). Additionally, the effect of hypoxia on DCs is very important in regulating the quality and intensity of the immune response. DCs derived from human monocytes exposed to hypoxia express high levels of HIF-1 $\alpha$. Short-term hypoxia can indeed enhance the migration of DCs through a HIF-1 $\alpha$-mediated glycolytic pathway, thus showing obvious immunogenicity. However, long-term hypoxia can cause cell death (113).

Of note, recent evidence has shown that the role of glucose in DCs depends on the state of adjacent cells (108). For example, glucose can promote the immune function of DCs, but this function is severely inhibited in areas of high ischemia and hypoxia in tumors (114). The reason is that the high rate of glycolysis of tumor cells leads to local glucose deprivation, and thus the substrate of glycolysis may not be used by DCs. Therefore, the TME may not be suitable for activation of DCs dependent on glycolysis, and thus DCs may be required to transition to fatty acid-dependent oxidation (8).

Important contribution of lipid metabolism to tolerant DCs. Herber et al (102) evaluated lipid accumulation in tumor-infiltrating DCs that would hinder antigen presentation and major histocompatibility complex II expression, and inhibition of acetyl-CoA carboxylase 1 reversed the effects of lipids, suggesting that this process involved the fatty acid biosynthesis pathway $(102,115)$. FASN is a key enzyme in the de novo synthesis of fatty acids. It directly supports the abilities of proliferation and metastasis in tumor cells $(116,117)$. A study reported that FASN was highly expressed in ovarian cancer cells, which led to lipid accumulation in the TME that inhibited the ability of tumor-infiltrated DCs to support antitumor T cells, resulting in antigen presentation that led to T cell activation defects (118).

DCs isolated from various tumor models and patients with tumors have shown that the accumulation of lipids in DCs limits the cross presentation of antigens (102). Consistent with these findings, the accumulation of lipid droplets prevents DCs from inducing antitumor T cell responses (119). DCs with an immunotolerant phenotype show strong activation of endoplasmic reticulum stress and X-box binding protein 1 spliced by endoplasmic reticulum stress response factor, which induces the biosynthesis of triglycerides and leads to abnormal lipid accumulation (103). The aforementioned studies suggest that lipid metabolism plays an important role in the development of tolerance in DCs.

\section{Other immune cells}

There are numerous types of infiltrated immune cells in the TME. In addition to the main immune cells mentioned above, there are also some immune cells that are less common, but still play important roles in antitumor immunity, such as mast cells, monocytes, eosinophils and basophils $(120,121)$. At present, the metabolic reprogramming of these cells in the TME is relatively unstudied. Some of these cell types have been studied briefly, while there are a few that have not yet been reported on, but they are worthy of further exploration. Mast cells are unique tissue-resident immune cells that can secrete a variety of bioactive compounds, which can stimulate, regulate or inhibit the immune response. Increasing evidence has reported that mast cells infiltrate breast cancer, but whether they are a driving force or have an opposing role in breast cancer progression is controversial (122-124).

In breast cancer, myeloid cells can transform into MDSCs and play a key role in tumor immunosuppression. Decades ago, studies demonstrated that cancer cells rather than stromal cells prefer to export fatty acids to form fatty acid-rich niches $(125,126)$. MDSCs absorb fatty acids from the TME and use these molecules in a variety of ways to maximize their effects. An increase in fatty acid uptake by MDSCs leads to the accumulation of intracellular lipids, and lipid-overloaded MDSCs have a stronger immunosuppressive effect on $\mathrm{CD}^{+} \mathrm{T}$ cells (127). Cao et al (128) revealed that the expression of fatty acid transporter 4 (FATP4) was significantly upregulated in MDSCs. Other studies have also 
Table II. Status of tumor-infiltrating immune cells under different metabolic patterns.

\begin{tabular}{|c|c|c|}
\hline Immune cells & Main metabolic modes & States \\
\hline \multirow[t]{3}{*}{ T lymphocytes } & Reduced nutrient transport and maintained catabolism & Primary \\
\hline & Glycolysis and lactic acid production & Activated \\
\hline & Lipolysis and lipid oxidation & Immunosuppressive \\
\hline \multirow[t]{2}{*}{ Macrophages } & $\begin{array}{l}\text { Enhanced aerobic glycolysis, pentose phosphate pathway activity and fatty acid } \\
\text { synthesis }\end{array}$ & M1 \\
\hline & Low levels of glycolysis and enhanced fatty acid oxidation & M2 \\
\hline \multirow[t]{3}{*}{ NK cells } & OXPHOS & Resting \\
\hline & Upregulated glycolysis, OXPHOS and lipid synthesis & Activated \\
\hline & Lipid metabolism & Inactivated \\
\hline \multirow[t]{2}{*}{ Dendritic cells } & Glycolysis & Immunostimulatory \\
\hline & OXPHOS and fatty acid oxidation & Immunosuppressive \\
\hline
\end{tabular}

OXPHOS, oxidative phosphorylation.

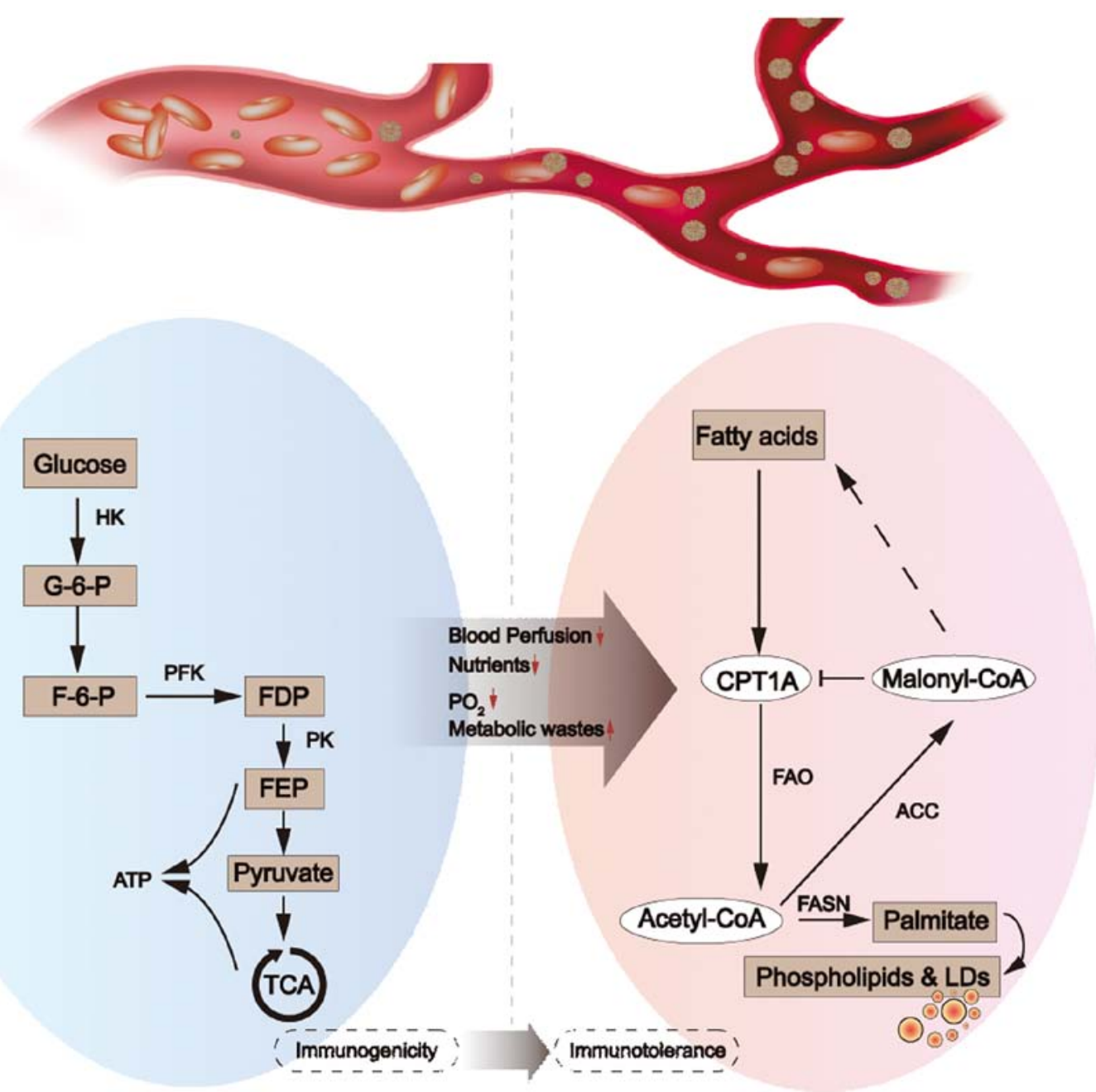

Figure 2. Priority of the immune cell metabolic mode is lipid metabolism during the transition from effector function to immune tolerance in the tumor microenvironment. Immune cells show corresponding effector functions with the metabolic mode of glycolysis. Glucose generates pyruvate through a series of reactions performed by the related enzymes, including HK, PFK and PK, and rapidly produces ATP and intermediate metabolites (G-6-P, F-6-P, FDP and PEP). However, immune cells are challenged by an environment comprising insufficient blood supply, decreased oxygen partial pressure, lack of nutrients and increased metabolic waste, which gradually leads to a tolerance phenotype, and the metabolic mode favors lipid metabolism, including FAO, and lipid synthesis (CPT1A, ACC, FASN, triglyceride and cholesterol). LDs play an important role in this process. On the one hand, LDs store excess lipids; on the other hand, LDs provide energy when needed. HK, hexokinase; PFK, phosphofructokinase; PK, pyruvate kinase; G-6-P, glucose-6-phosphate; F-6-P, fructose 6-phosphate; FDP, fructose 1,6-bisphosphate; PEP, phosphoenolpyruvate; FAO, fatty acid oxidation; CPT1A, carnitine palmitoyl transferase 1A; ACC, acetyl CoA carboxylase; FASN, fatty acid synthase; LDs, lipid droplets; TCA, trichloroacetic acid. 
reported that the upregulation of FATP2 expression in polymorphonuclear myeloid-derived suppressor cells (PMN-MDSCs) is the key regulator of PMN-MDSC immunosuppressive function. FATP2 promotes the accumulation of arachidonic acid, resulting in the synthesis of prostaglandin E2 in MDSCs, which enhances MDSC immunosuppressive activity (129). A series of changes lead to MDSCs surviving in the harsh TME. The role of lipid metabolism in determining whether immune cells differentiate into an immunotolerant phenotype has been ignored, as has whether immunosuppressive cell infiltration of tumor tissue is induced by a harsh environment, which provide novel ideas and insights for tumor immune research.

\section{Conclusions}

The complex and dynamic changes in the TME cause infiltrating immune cells to face different challenges. Compared with the randomness and chaos of genetic changes, predictable extracellular metabolite gradients allow speculation on the metabolic changes experienced by immune cells. By studying and summarizing the changes in the metabolic patterns of several typical tumor-infiltrating immune cell types in different TMEs (Table II), the present review concluded that mild hypoxia and a low lactic acid concentration are beneficial for immune cell activation and a metabolic mode favoring glycolysis. In such a scenario, the immune cells have certain immunogenicity and play an antitumor role. However, facing severe survival challenges, such as hypoxia, an extreme lack of nutrients and high acidity in the surrounding environment, the immune cells will gradually shift their metabolism mode into lipid metabolism, in some cases, even depending on FAO, and the immune cells will show a tolerant or inhibited phenotype (Fig. 2). The function of immune cells also changes when the metabolism mode changes, which provides us with a number of potential immunotherapy targets and novel treatment ideas. If the key genes and enzymes of lipid metabolism can be targeted, it may be possible to reverse the tolerant phenotype of immune cells and enhance the antitumor effect. In the future, it is necessary to study the metabolic heterogeneity of immune cells and the causes of this heterogeneity, in order to provide more effective methods for tumor immunotherapy.

\section{Acknowledgements}

Not applicable.

\section{Funding}

This work was supported by the Natural Science Foundation Project of Chongqing, CSTC (grant nos. cstc2020jcyj-msxmX 0485 and cstc2020jcyj-msxmX0668).

\section{Availability of data and materials}

Not applicable.

\section{Authors' contributions}

HC, YS, ZY, SY, YL, MT, JZ and FZ contributed to the conceptualization, literature review, original draft preparation, editing and review of this manuscript. All authors read and approved the final manuscript.

\section{Ethics approval and consent to participate}

Not applicable.

\section{Patient consent for publication}

Not applicable.

\section{Competing interests}

The authors declare that they have no competing interests.

\section{References}

1. Siegel RL, Miller KD and Jemal A: Cancer statistics, 2019. CA Cancer J Clin 69: 7-34, 2019.

2. Vranic S, Cyprian FS, Gatalica Z and Palazzo J: PD-L1 status in breast cancer: Current view and perspectives. Semin Cancer Biol: Dec 26, 2019 (Epub ahead of print).

3. Engelhard VH, Rodriguez AB, Mauldin IS, Woods AN, Peske JD and Slingluff CL Jr: Immune Cell Infiltration and tertiary lymphoid structures as determinants of antitumor immunity. J Immunol 200: 432-442, 2018.

4. Savas P, Virassamy B, Ye C, Salim A, Mintoff CP, Caramia F, Salgado R, Byrne DJ, Teo ZL, Dushyanthen S, et al: Publisher correction: Single-cell profiling of breast cancer T cells reveals a tissue-resident memory subset associated with improved prognosis. Nat Med 24: 1941, 2018.

5. Zhang SC, Hu ZQ, Long JH, Zhu GM, Wang Y, Jia Y, Zhou J, Ouyang Y and Zeng Z: Clinical implications of tumor-infiltrating immune cells in breast cancer. J Cancer 10: 6175-6184, 2019.

6. Wu D: Innate and adaptive immune cell metabolism in tumor microenvironment. Adv Exp Med Biol 1011: 211-223, 2017.

7. Kishton RJ, Sukumar M and Restifo NP: Metabolic regulation of $\mathrm{T}$ cell longevity and function in tumor immunotherapy. Cell Metab 26: 94-109, 2017.

8. Hobson-Gutierrez SA and Carmona-Fontaine C: The metabolic axis of macrophage and immune cell polarization. Dis Model Mech 11: dmm034462, 2018.

9. Warburg O: Über den Stoffwechsel der Carcinomzelle. Naturwissenschaften 12: 1131-1137, 1924.

10. Lane AN, Higashi RM and Fan TW: Metabolic reprogramming in tumors: Contributions of the tumor microenvironment. Genes Dis 7: 185-198, 2019.

11. Kim J and DeBerardinis RJ: Mechanisms and implications of metabolic heterogeneity in cancer. Cell Metab 30: 434-446, 2019.

12. Mehla K and Singh PK: Metabolic regulation of macrophage polarization in cancer. Trends Cancer 5: 822-834, 2019.

13. Basit F, Mathan T, Sancho D and de Vries IJM: Human dendritic cell subsets undergo distinct metabolic reprogramming for immune response. Front Immunol 9: 2489, 2018.

14. Zhang L and Romero P: Metabolic control of CD8(+) T cell fate decisions and antitumor immunity. Trends Mol Med 24: 30-48, 2018.

15. Poznanski SM, Barra NG, Ashkar AA and Schertzer JD: Immunometabolism of T cells and NK cells: Metabolic control of effector and regulatory function. Inflamm Res 67: 813-828, 2018.

16. Norton KA, Jin K and Popel AS: Modeling triple-negative breast cancer heterogeneity: Effects of stromal macrophages, fibroblasts and tumor vasculature. J Theor Biol 452: 56-68, 2018.

17. Skala MC, Fontanella A, Lan L, Izatt JA and Dewhirst MW: Longitudinal optical imaging of tumor metabolism and hemodynamics. J Biomed Opt 15: 011112, 2010.

18. Roulot A, Héquet D, Guinebretière JM, Vincent-Salomon A, Lerebours F, Dubot C and Rouzier R: Tumoral heterogeneity of breast cancer. Ann Biol Clin (Paris) 74: 653-660, 2016.

19. Carmona-Fontaine C, Deforet M, Akkari L, Thompson CB, Joyce JA and Xavier JB: Metabolic origins of spatial organization in the tumor microenvironment. Proc Natl Acad Sci USA 114: 2934-2939, 2017 
20. Montcourrier P, Silver I, Farnoud R, Bird I and Rochefort H: Breast cancer cells have a high capacity to acidify extracellular milieu by a dual mechanism. Clin Exp Metastasis 15: 382-392, 1997.

21. Logozzi M, Spugnini E, Mizzoni D, Di Raimo R and Fais S: Extracellular acidity and increased exosome release as key phenotypes of malignant tumors. Cancer Metastasis Rev 38 93-101, 2019.

22. Gao T, Li JZ, Lu Y, Zhang CY,Li Q, Mao J and Li LH: The mechanism between epithelial mesenchymal transition in breast cancer and hypoxia microenvironment. Biomed Pharmacother 80 393-405, 2016

23. Liu ZJ, Semenza GL and Zhang HF: Hypoxia-inducible factor 1 and breast cancer metastasis. J Zhejiang Univ Sci B 16: 32-43, 2015.

24. Daşu A, Toma-Daşu I and Karlsson M: Theoretical simulation of tumour oxygenation and results from acute and chronic hypoxia. Phys Med Biol 48: 2829-2842, 2003.

25. Mantovani A, Allavena P, Sica A and Balkwill F: Cancer-related inflammation. Nature 454: 436-444, 2008.

26. Byrne A, Savas P, Sant S, Li R, Virassamy B, Luen SJ, Beavis PA, Mackay LK, Neeson PJ and Loi S: Tissue-resident memory T cells in breast cancer control and immunotherapy responses. Nat Rev Clin Oncol 17: 341-348, 2020.

27. Frauwirth KA, Riley JL, Harris MH, Parry RV, Rathmell JC, Plas DR, Elstrom RL, June CH and Thompson CB: The CD28 signaling pathway regulates glucose metabolism. Immunity 16 : 769-777, 2002

28. Kamiński MM, Sauer SW, Kamiński M, Opp S, Ruppert T, Grigaravičius P, Grudnik P, Gröne HJ, Krammer PH and Gülow K $\mathrm{T}$ cell activation is driven by an ADP-dependent glucokinase linking enhanced glycolysis with mitochondrial reactive oxygen species generation. Cell Rep 2: 1300-1315, 2012.

29. Gentric G, Mieulet V and Mechta-Grigoriou F: Heterogeneity in cancer metabolism: New concepts in an old field. Antioxid Redox Signal 26: 462-485, 2017.

30. MacPherson S, Kilgour M and Lum JJ: Understanding lymphocyte metabolism for use in cancer immunotherapy. FEBS J 285 $2567-2578,2018$

31. Michalek RD, Gerriets VA, Jacobs SR, Macintyre AN MacIver NJ, Mason EF, Sullivan SA, Nichols AG and Rathmell JC: Cutting edge: Distinct glycolytic and lipid oxidative metabolic programs are essential for effector and regulatory CD4+ T cell subsets. J Immunol 186: 3299-3303, 2011.

32. Berod L, Friedrich C, Nandan A, Freitag J, Hagemann S, Harmrolfs K, Sandouk A, Hesse C, Castro CN, Bähre H, et al: De novo fatty acid synthesis controls the fate between regulatory T and T helper 17 cells. Nat Med 20: 1327-1333, 2014.

33. Phan AT and Goldrath AW: Hypoxia-inducible factors regulate T cell metabolism and function. Mol Immunol 68: 527-535, 2015

34. Kim JW, Tchernyshyov I, Semenza GL and Dang CV: HIF-1-mediated expression of pyruvate dehydrogenase kinase: A metabolic switch required for cellular adaptation to hypoxia. Cell Metab 3: 177-185, 2006

35. Westendorf AM, Skibbe K, Adamczyk A, Buer J, Geffers R, Hansen W, Pastille E and Jendrossek V: Hypoxia enhances immunosuppression by inhibiting CD4+ effector T cell function and promoting treg activity. Cell Physiol Biochem 41: 1271-1284, 2017.

36. Molon B, Calì B and Viola A: T cells and cancer: How metabolism shapes immunity. Front Immunol 7: 20, 2016.

37. Mack N, Mazzio EA, Bauer D, Flores-Rozas H and Soliman KF: Stable shRNA silencing of lactate dehydrogenase A (LDHA) in human MDA-MB-231 breast cancer cells fails to alter lactic acid production, glycolytic activity, ATP or survival. Anticancer Res 37: 1205-1212, 2017.

38. Siska PJ and Rathmell JC: T cell metabolic fitness in antitumor immunity. Trends Immunol 36: 257-264, 2015.

39. Peppicelli S, Toti A, Giannoni E, Bianchini F, Margheri F, Del Rosso M and Calorini L: Metformin is also effective on lactic acidosis-exposed melanoma cells switched to oxidative phosphorylation. Cell Cycle 15: 1908-1918, 2016.

40. Fischer K, Hoffmann P, Voelkl S, Meidenbauer N, Ammer J, Edinger M, Gottfried E, Schwarz S, Rothe G, Hoves S, et al: Inhibitory effect of tumor cell-derived lactic acid on human $\mathrm{T}$ cells. Blood 109: 3812-3819, 2007.

41. Ho PC, Bihuniak JD, Macintyre AN, Staron M, Liu X, Amezquita R, Tsui YC, Cui G, Micevic G, Perales JC, et al: Phosphoenolpyruvate Is a metabolic checkpoint of anti-tumor $\mathrm{t}$ cell responses. Cell 162: 1217-1228, 2015.
42. Beckermann KE, Dudzinski SO and Rathmell JC: Dysfunctional $\mathrm{T}$ cell metabolism in the tumor microenvironment. Cytokine Growth Factor Rev 35: 7-14, 2017.

43. Neugent ML, Goodwin J, Sankaranarayanan I, Yetkin CE, Hsieh MH and Kim JW: A new perspective on the heterogeneity of cancer glycolysis. Biomol Ther (Seoul) 26: 10-18, 2018.

44. Angelin A, Gil-de-Gómez L, Dahiya S, Jiao J, Guo L, Levine MH, Wang Z, Quinn WJ III, Kopinski PK, Wang L, et al: Foxp3 reprograms $\mathrm{T}$ cell metabolism to function in low-glucose high-lactate environments. Cell Metab 25: 1282-1293.e7, 2017.

45. Hao Y, Li D, Xu Y, Ouyang J, Wang Y, Zhang Y, Li B, Xie L and Qin G: Investigation of lipid metabolism dysregulation and the effects on immune microenvironments in pan-cancer using multiple omics data. BMC Bioinformatics 20 (Suppl 7): S195, 2019.

46. Saleh $\mathrm{R}$ and Elkord E: FoxP3 ${ }^{+} \mathrm{T}$ regulatory cells in cancer: Prognostic biomarkers and therapeutic targets. Cancer Lett 490: 174-185, 2020

47. Iranparast S, Tayebi S, Ahmadpour F and Yousefi B Tumor-Induced metabolism and $\mathrm{T}$ cells located in tumor environment. Curr Cancer Drug Targets 20: 741-756, 2020.

48. Shang $\mathrm{W}, \mathrm{Xu} \mathrm{R}, \mathrm{Xu} \mathrm{T}, \mathrm{Wu} \mathrm{M}, \mathrm{Xu} \mathrm{J}$ and Wang F: Ovarian cancer cells promote glycolysis metabolism and TLR8-mediated metabolic control of human CD4+ T cells. Front Oncol 10: 570899, 2020.

49. Vardhana SA, Hwee MA, Berisa M, Wells DK, Yost KE, King B, Smith M, Herrera PS, Chang HY, Satpathy AT, et al: Impaired mitochondrial oxidative phosphorylation limits the self-renewal of $\mathrm{T}$ cells exposed to persistent antigen. Nat Immunol 21: $1022-1033,2020$

50. Beier UH, Angelin A, Akimova T, Wang L, Liu Y, Xiao H, Koike MA, Hancock SA, Bhatti TR, Han R, et al: Essential role of mitochondrial energy metabolism in Foxp3+ T-regulatory cell function and allograft survival. FASEB J 29: 2315-2326, 2015.

51. Gerriets VA, Kishton RJ, Nichols AG, Macintyre AN, Inoue M, Ilkayeva O, Winter PS, Liu X, Priyadharshini B, Slawinska ME, et al: Metabolic programming and PDHK1 control CD $4^{+} \mathrm{T}$ cell subsets and inflammation. J Clin Invest 125 : 194-207, 2015

52. Duan W, Ding Y, Yu X, Ma D, Yang B, Li Y, Huang L, Chen Z, Zheng $\mathrm{J}$ and Yang $\mathrm{C}$ : Metformin mitigates autoimmune insulitis by inhibiting Th1 and Th17 responses while promoting Treg production. Am J Transl Res 11: 2393-2402, 2019.

53. Lu L, Barbi J and Pan F: The regulation of immune tolerance by FOXP3. Nat Rev Immunol 17: 703-717, 2017.

54. Georgiev P, Charbonnier LM and Chatila TA: Regulatory T cells: The many faces of Foxp3. J Clin Immunol 39: 623-640, 2019.

55. Gerriets VA, Kishton RJ, Johnson MO, Cohen S, Siska PJ, Nichols AG, Warmoes MO, de Cubas AA, MacIver NJ, Locasale JW, et al: Foxp3 and Toll-like receptor signaling balance $\mathrm{T}_{\mathrm{reg}}$ cell anabolic metabolism for suppression. Nat Immunol 17: 1459-1466, 2016.

56. Chen X, Feng L, Li S, Long D, Shan J and Li Y: TGF- $\beta 1$ maintains Foxp3 expression and inhibits glycolysis in natural regulatory $\mathrm{T}$ cells via PP2A-mediated suppression of mTOR signaling. Immunol Lett 226: 31-37, 2020.

57. Choi J, Gyamfi J, Jang H and Koo JS: The role of tumor-associated macrophage in breast cancer biology. Histol Histopathol 33 133-145, 2018.

58. Su S, Liu Q, Chen J, Chen J, Chen F, He C, Huang D, Wu W, Lin L, Huang W, et al: A positive feedback loop between mesenchymal-like cancer cells and macrophages is essential to breast cancer metastasis. Cancer Cell 25: 605-620, 2014.

59. Klingen TA, Chen Y, Aas H, Wik E and Akslen LA: Tumor-associated macrophages are strongly related to vascular invasion, non-luminal subtypes, and interval breast cancer. Hum Pathol 69: 72-80, 2017.

60. Tarique AA, Logan J, Thomas E, Holt PG, Sly PD and Fantino E: Phenotypic, functional, and plasticity features of classical and alternatively activated human macrophages. Am J Respir Cell Mol Biol 53: 676-688, 2015.

61. Geeraerts X, Bolli E, Fendt SM and Van Ginderachter JA: Macrophage metabolism as therapeutic target for cancer, atherosclerosis, and obesity. Front Immunol 8: 289, 2017.

62. Mantovani A, Marchesi F, Malesci A, Laghi L and Allavena P: Tumour-associated macrophages as treatment targets in oncology. Nat Rev Clin Oncol 14: 399-416, 2017.

63. De Santa F, Vitiello L, Torcinaro A and Ferraro E: The role of metabolic remodeling in macrophage polarization and its effect on skeletal muscle regeneration. Antioxid Redox Signal 30: 1553-1598, 2019 
64. Van den Bossche J, Baardman J, Otto NA, van der Velden S, Neele AE, van den Berg SM, Luque-Martin R, Chen HJ, Boshuizen MC, Ahmed M, et al: Mitochondrial dysfunction prevents repolarization of inflammatory macrophages. Cell Rep 17: 684-696, 2016.

65. Kim J: Regulation of immune cell functions by metabolic reprogramming. J Immuno Res 2018: 8605471, 2018.

66. Rodríguez-Prados JC, Través PG, Cuenca J, Rico D, Aragonés J, Martín-Sanz P, Cascante M and Boscá L: Substrate fate in activated macrophages: A comparison between innate, classic, and alternative activation. J Immunol 185: 605-614, 2010.

67. Vats D, Mukundan L, Odegaard JI, Zhang L, Smith KL, Morel CR, Wagner RA, Greaves DR, Murray PJ and Chawla A: Oxidative metabolism and PGC-1beta attenuate macrophage-mediated inflammation. Cell Metab 4: 13-24, 2006.

68. Colegio OR, Chu NQ, Szabo AL, Chu T, Rhebergen AM, Jairam V, Cyrus N, Brokowski CE, Eisenbarth SC, Phillips GM, et al: Functional polarization of tumour-associated macrophages by tumour-derived lactic acid. Nature 513: 559-563, 2014.

69. Feng R, Morine Y, Ikemoto T, Imura S, Iwahashi S, Saito Y and Shimada M: Nrf2 activation drive macrophages polarization and cancer cell epithelial-mesenchymal transition during interaction. Cell Commun Signal 16: 54, 2018.

70. Maftouh M, Avan A, Sciarrillo R, Granchi C, Leon LG, Rani R, Funel N, Smid K, Honeywell R, Boggi U, et al: Synergistic interaction of novel lactate dehydrogenase inhibitors with gemcitabine against pancreatic cancer cells in hypoxia. $\mathrm{Br}$ J Cancer 110: 172-182, 2014

71. Mediani L, Gibellini F, Bertacchini J, Frasson C, Bosco R, Accordi B, Basso G, Bonora M, Calabrò ML, Mattiolo A, et al: Reversal of the glycolytic phenotype of primary effusion lymphoma cells by combined targeting of cellular metabolism and PI3K/Akt/mTOR signaling. Oncotarget 7: 5521-5537, 2016

72. Lin S, Sun L, Lyu X, Ai X, Du D, Su N, Li H, Zhang L, Yu J and Yuan S: Lactate-activated macrophages induced aerobic glycolysis and epithelial-mesenchymal transition in breast cancer by regulation of CCL5-CCR5 axis: A positive metabolic feedback loop. Oncotarget 8: 110426-110443, 2017.

73. Viola A, Munari F, Sánchez-Rodríguez R, Scolaro T and Castegna A: The metabolic signature of macrophage responses. Front Immunol 10: 1462, 2019.

74. Zhang Q, Wang H, Mao C, Sun M, Dominah G, Chen L and Zhuang Z: Fatty acid oxidation contributes to IL-1 $\beta$ secretion in M2 macrophages and promotes macrophage-mediated tumor cell migration. Mol Immunol 94: 27-35, 2018.

75. Wu H, Han Y, Rodriguez Sillke Y, Deng H, Siddiqui S, Treese C, Schmidt F, Friedrich M, Keye J, Wan J, et al: Lipid droplet-dependent fatty acid metabolism controls the immune suppressive phenotype of tumor-associated macrophages. EMBO Mol Med 11: e10698, 2019.

76. Rombaldova M, Janovska P, Kopecky J and Kuda O: Omega-3 fatty acids promote fatty acid utilization and production of pro-resolving lipid mediators in alternatively activated adipose tissue macrophages. Biochem Biophys Res Commun 490 1080-1085, 2017.

77. Huang SC, Everts B, Ivanova Y, O'Sullivan D, Nascimento M, Smith AM, Beatty W, Love-Gregory L, Lam WY O'Neill CM, et al: Cell-intrinsic lysosomal lipolysis is essential for alternative activation of macrophages. Nat Immunol 15: 846-855, 2014.

78. Jin $\mathrm{H}$, He Y, Zhao P, Hu Y, Tao J, Chen J and Huang Y: Targeting lipid metabolism to overcome EMT-associated drug resistance via integrin $\beta 3 /$ FAK pathway and tumor-associated macrophage repolarization using legumain-activatable delivery. Theranostics 9: 265-278, 2019.

79. Chiossone L, Dumas PY, Vienne M and Vivier E: Natural killer cells and other innate lymphoid cells in cancer. Nat Rev Immunol 18: 671-688, 2018

80. Terrén I, Orrantia A, Vitallé J, Zenarruzabeitia $\mathrm{O}$ and Borrego $\mathrm{F}$ : NK cell metabolism and tumor microenvironment. Front Immunol 10: 2278, 2019.

81. Gardiner CM: NK cell metabolism. J Leukoc Biol 105 1235-1242, 2019

82. Keating SE, Zaiatz-Bittencourt V, Loftus RM, Keane C, Brennan K, Finlay DK and Gardiner CM: Metabolic reprogramming supports IFN $-\gamma$ production by CD56bright NK cells. J Immunol 196: 2552-2560, 2016.
83. Keppel MP, Saucier N, Mah AY, Vogel TP and Cooper MA: Activation-specific metabolic requirements for NK Cell IFN- $\gamma$ production. J Immunol 194: 1954-1962, 2015.

84. Assmann N, O'Brien KL, Donnelly RP, Dyck L, Zaiatz-Bittencourt V, Loftus RM, Heinrich P, Oefner PJ, Lynch L, Gardiner CM, et al: Srebp-controlled glucose metabolism is essential for NK cell functional responses. Nat Immunol 18: 1197-1206, 2017.

85. Cooper MA, Fehniger TA and Caligiuri MA: The biology of human natural killer-cell subsets. Trends Immunol 22: 633-640, 2001

86. Schafer JR, Salzillo TC, Chakravarti N, Kararoudi MN, Trikha P, Foltz JA, Wang R, Li S and Lee DA: Education-dependent activation of glycolysis promotes the cytolytic potency of licensed human natural killer cells. J Allergy Clin Immunol 143: 346-358.e6, 2019.

87. Parodi M, Raggi F, Cangelosi D, Manzini C, Balsamo M, Blengio F, Eva A, Varesio L, Pietra G, Moretta L, et al: Hypoxia modifies the transcriptome of human NK cells, modulates their immunoregulatory profile, and influences NK cell subset migration. Front Immunol 9: 2358, 2018.

88. Balsamo M, Manzini C, Pietra G, Raggi F, Blengio F, Mingari MC, Varesio L, Moretta L, Bosco MC and Vitale M: Hypoxia downregulates the expression of activating receptors involved in NK-cell-mediated target cell killing without affecting ADCC. Eur J Immunol 43: 2756-2764, 2013.

89. Dengler VL, Galbraith M and Espinosa JM: Transcriptional regulation by hypoxia inducible factors. Crit Rev Biochem Mol Biol 49: 1-15, 2014.

90. Yang C, Tsaih SW, Lemke A, Flister MJ, Thakar MS and Malarkannan S: mTORC1 and mTORC2 differentially promote natural killer cell development. Elife 7: e35619, 2018.

91. Chambers AM, Wang J, Lupo KB, Yu H, Atallah Lanman NM and Matosevic S: Adenosinergic signaling alters natural killer cell functional responses. Front Immunol 9: 2533, 2018.

92. Brand A, Singer K, Koehl GE, Kolitzus M, Schoenhammer G, Thiel A, Matos C, Bruss C, Klobuch S, Peter K, et al: LDHA-associated lactic acid production blunts tumor immunosurveillance by T and NK cells. Cell Metab 24: 657-671, 2016.

93. Stiff A, Trikha P, Mundy-Bosse B, McMichael E, Mace TA, Benner B, Kendra K, Campbell A, Gautam S, Abood D, et al: Nitric oxide production by myeloid-derived suppressor cells plays a role in impairing $\mathrm{Fc}$ receptor-mediated natural killer cell function. Clin Cancer Res 24: 1891-1904, 2018.

94. Piñeiro Fernández J, Luddy KA, Harmon C and O'Farrelly C: Hepatic tumor microenvironments and effects on $\mathrm{NK}$ cell phenotype and function. Int J Mol Sci 20: 4131, 2019.

95. Vitale M, Cantoni C, Pietra G, Mingari MC and Moretta L Effect of tumor cells and tumor microenvironment on NK-cell function. Eur J Immunol 44: 1582-1592, 2014.

96. Wang Z, Guan D, Wang S, Chai LYA, Xu S and Lam KP: Glycolysis and oxidative phosphorylation play critical roles in natural killer cell receptor-mediated natural killer cell functions. Front Immunol 11: 202, 2020.

97. Terrén I,Orrantia A, Vitallé J,Astarloa-Pando G,ZenarruzabeitiaO and Borrego F: Modulating NK cell metabolism for cancer immunotherapy. Semin Hematol 57: 213-224, 2020.

98. Kobayashi T, Lam PY, Jiang H, Bednarska K, Gloury RE, Murigneux V, Tay J, Jacquelot N, Li R, Tuong ZK, et al: Increased lipid metabolism impairs NK cell function and mediates adaptation to the lymphoma environment. Blood: Aug 20, 2020 (Epub ahead of print).

99. Inoue H, Miyaji M, Kosugi A, Nagafuku M, Okazaki T, Mimori T, Amakawa R, Fukuhara S, Domae N, Bloom ET and Umehara $\mathrm{H}$ : Lipid rafts as the signaling scaffold for NK cell activation: Tyrosine phosphorylation and association of LAT with phosphatidylinositol 3-kinase and phospholipase C-gamma following CD2 stimulation. Eur J Immunol 32: 2188-2198, 2002.

100. Niavarani SR, Lawson C, Bakos O, Boudaud M, Batenchuk C, Rouleau S and Tai LH: Lipid accumulation impairs natural killer cell cytotoxicity and tumor control in the postoperative period. BMC Cancer 19: 823, 2019.

101. Michelet X, Dyck L, Hogan A, Loftus RM, Duquette D, Wei K, Beyaz S, Tavakkoli A, Foley C, Donnelly R, et al: Metabolic reprogramming of natural killer cells in obesity limits antitumor responses. Nat Immunol 19: 1330-1340, 2018.

102. Herber DL, Cao W, Nefedova Y, Novitskiy SV, Nagaraj S, Tyurin VA, Corzo A, Cho HI, Celis E, Lennox B, et al: Lipid accumulation and dendritic cell dysfunction in cancer. Nat Med 16: 880-886, 2010. 
103. Gao F, Liu C, Guo J, Sun W, Xian L, Bai D, Liu H, Cheng Y, Li B, Cui J, et al: Radiation-driven lipid accumulation and dendritic cell dysfunction in cancer. Sci Rep 5: 9613, 2015.

104. Dong $\mathrm{H}$ and Bullock TN: Metabolic influences that regulate dendritic cell function in tumors. Front Immunol 5: 24, 2014.

105. Brown TP, Bhattacharjee P, Ramachandran S, Sivaprakasam S, Ristic B, Sikder MOF and Ganapathy V: The lactate receptor GPR81 promotes breast cancer growth via a paracrine mechanism involving antigen-presenting cells in the tumor microenvironment. Oncogene 39: 3292-3304, 2020.

106. Ibrahim J, Nguyen AH, Rehman A, Ochi A, Jamal M, Graffeo CS, Henning JR, Zambirinis CP, Fallon NC, Barilla R, et al: Dendritic cell populations with different concentrations of lipid regulate tolerance and immunity in mouse and human liver. Gastroenterology 143: 1061-1072, 2012.

107. Mellor AL and Munn DH: Creating immune privilege: Active local suppression that benefits friends, but protects foes. Nat Rev Immunol 8: 74-80, 2008

108. Everts B, Amiel E, Huang SC, Smith AM, Chang CH, Lam WY, Redmann V, Freitas TC, Blagih J, van der Windt GJ, et al: TLR-driven early glycolytic reprogramming via the kinases TBK1-IKKe supports the anabolic demands of dendritic cell activation. Nat Immunol 15: 323-332, 2014.

109. Guak H, Al Habyan S, Ma EH, Aldossary H, Al-Masri M, Won SY, Ying T, Fixman ED, Jones RG, McCaffrey LM and Krawczyk CM: Glycolytic metabolism is essential for CCR7 oligomerization and dendritic cell migration. Nat Commun 9: $2463,2018$.

110. Krawczyk CM, Holowka T, Sun J, Blagih J, Amiel E, DeBerardinis RJ, Cross JR, Jung E, Thompson CB, Jones RG and Pearce EJ: Toll-like receptor-induced changes in glycolytic metabolism regulate dendritic cell activation. Blood 115: 4742-4749, 2010.

111. Nasi A, Fekete T, Krishnamurthy A, Snowden S, Rajnavölgyi E, Catrina AI, Wheelock CE, Vivar N and Rethi B: Dendritic cell reprogramming by endogenously produced lactic acid. J Immunol 191: 3090-3099, 2013.

112. Gottfried E, Kunz-Schughart LA, Ebner S, Mueller-Klieser W, Hoves S, Andreesen R, Mackensen A and Kreutz M: Tumor-derived lactic acid modulates dendritic cell activation and antigen expression. Blood 107: 2013-2021, 2006.

113. Naldini A, Morena E, Pucci A, Miglietta D, Riboldi E, Sozzani S and Carraro F: Hypoxia affects dendritic cell survival: Role of the hypoxia-inducible factor-1 $\alpha$ and lipopolysaccharide. J Cell Physiol 227: 587-595, 2012.

114. Lawless SJ, Kedia-Mehta N, Walls JF, McGarrigle R, Convery O, Sinclair LV, Navarro MN, Murray J and Finlay DK: Glucose represses dendritic cell-induced T cell responses. Nat Commun 8: 15620, 2017.

115. Ramakrishnan R, Tyurin VA, Veglia F, Condamine $T$, Amoscato A, Mohammadyani D, Johnson JJ, Zhang LM, Klein-Seetharaman J, Celis E, et al: Oxidized lipids block antigen cross-presentation by dendritic cells in cancer. J Immunol 192: 2920-2931, 2014.

116. Menendez JA and Lupu R: Fatty acid synthase (FASN) as a therapeutic target in breast cancer. Expert Opin Thera Targets 21: 1001-1016, 2017.
117. Ventura R, Mordec K, Waszczuk J, Wang Z, Lai J, Fridlib M, Buckley D, Kemble G and Heuer TS: Inhibition of de novo palmitate synthesis by fatty acid synthase induces apoptosis in tumor cells by remodeling cell membranes, inhibiting signaling pathways, and reprogramming gene expression. EBioMedicine 2: 808-824, 2015.

118. Jiang L, Fang X, Wang H, Li D and Wang X: Ovarian cancer-intrinsic fatty acid synthase prevents anti-tumor immunity by disrupting tumor-infiltrating dendritic cells. Front Immunol 9: 2927, 2018.

119. Cubillos-Ruiz JR, Silberman PC, Rutkowski MR, Chopra S, Perales-Puchalt A, Song M, Zhang S, Bettigole SE, Gupta D, Holcomb K, et al: ER Stress Sensor XBP1 controls Anti-tumor immunity by disrupting dendritic cell homeostasis. Cell 161: $1527-1538,2015$.

120. Xiong Y, Liu L, Xia Y, Qi Y, Chen Y, Chen L, Zhang P, Kong Y, Qu Y, Wang Z, et al: Tumor infiltrating mast cells determine oncogenic HIF- $2 \alpha$-conferred immune evasion in clear cell renal cell carcinoma. Cancer Immunol Immunother 68: 731-741, 2019.

121. Schwartz M,Zhang Y and Rosenblatt JD: B cell regulation of the anti-tumor response and role in carcinogenesis. J Immunother Cancer 4: 40, 2016.

122. Aponte-López A, Fuentes-Pananá EM, Cortes-Muñoz D and Muñoz-Cruz S: Mast cell, the neglected member of the tumor microenvironment: Role in breast cancer. J Immunol Res 2018: 2584243, 2018.

123. Okano M, Oshi M, Butash AL, Katsuta E, Tachibana K, Saito K, Okayama H, Peng X, Yan L, Kono K, Ohtake T and Takabe K: Triple-negative breast cancer with high levels of Annexin A1 expression is associated with mast cell infiltration, inflammation, and angiogenesis. Int J Mol Sci 20: 4197, 2019.

124. Glajcar A, Szpor J, Pacek A, Tyrak KE, Chan F, Streb J, Hodorowicz-Zaniewska D and Okoń K: The relationship between breast cancer molecular subtypes and mast cell populations in tumor microenvironment. Virchows Arch 470: 505-515, 2017.

125. Spector AA: The importance of free fatty acid in tumor nutrition. Cancer Res 27: 1580-1586, 1967.

126. Li Z and Zhang H: Reprogramming of glucose, fatty acid and amino acid metabolism for cancer progression. Cell Mol Life Sci 73: 377-392, 2016.

127. Al-Khami AA,Zheng L, Del Valle L, Hossain F, Wyczechowska D, Zabaleta J, Sanchez MD, Dean MJ, Rodriguez PC and Ochoa AC: Exogenous lipid uptake induces metabolic and functional reprogramming of tumor-associated myeloid-derived suppressor cells. Oncoimmunology 6: e1344804, 2017.

128. Cao W and Gabrilovich D: Abstract 3649: Contribution of fatty acid accumulation to myeloid-derived suppressor cell function in cancer. Cancer Res 71: 3649-3649, 2011.

129. Veglia F, Tyurin VA, Blasi M, De Leo A, Kossenkov AV, Donthireddy L, To TKJ, Schug Z, Basu S, Wang F, et al: Fatty acid transport protein 2 reprograms neutrophils in cancer. Nature 569: 73-78, 2019.

This work is licensed under a Creative Commons Attribution-NonCommercial-NoDerivatives 4.0 International (CC BY-NC-ND 4.0) License. 